\title{
Metabolic noise reduction for enzymatic reactions: the role of a negative feedback
}

\author{
Alessandro Borri Pasquale Palumbo Abhyudai Singh
}

\begin{abstract}
This note deals with a basic (though rather general) enzymatic reaction scheme, and investigates the role of a negative feedback with respect to the noise reduction. To this end, three distinct cases are considered: one with the enzyme produced without feedback regulation, another with the enzyme regulated in feedback by the product of the enzymatic reaction, and a third one where the enzyme is self-regulated. Metabolic noise is evaluated in terms of the coefficient of variation of the product of the enzymatic reaction, aiming at measuring its fluctuations around the average steady-state value. Due to the high dimensionality and to the double time-scale of the considered reaction network, which makes unfeasible the classical theoretical/statistical computations of the equilibrium distribution, the system is investigated by means of the Linear Noise Approximation (LNA) approach and numerical results are reported. Furthermore, the assumption of Quasi-SteadyState Approximation (QSSA) is employed to obtain approximate analytical expressions of the noise. The results show the unmistakable role of the negative feedback, which always manages to reduce the metabolic noise with respect to the unregulated case.
\end{abstract}

Index Terms - Enzymatic reactions, Chemical Master Equation, Linear Noise Approximation, Negative Feedback

\section{INTRODUCTION}

Enzymatic reactions have been widely studied since the beginning of the last century [1], [2], and nowadays many fruitful approaches can be exploited to investigate their behavior according to deterministic (e.g. by means of Ordinary Differential Equations, ODEs, aiming at catching the average behavior) or stochastic approaches (e.g. detailing the random molecular fluctuations by means of the Chemical Master Equations, CMEs).

This note investigates the role of the feedback in the enzymatic production rate for a basic (though rather general) reaction scheme, aiming at quantifying the level of noise reduction (if any) with respect to the fluctuations of the final product of the enzymatic reaction. The role of the feedback in Systems Biology has been widely investigated, especially in transcriptional and metabolic regulation where gene products are required to control their homeostatic levels robustly with respect to parameter or environmental fluctuations [3], [4], [5].

A. Borri and P. Palumbo are with IASI-CNR, Italian National Research Council, Rome, Italy. E-mail addresses: \{alessandro.borri, pasquale.palumbo\}@iasi.cnr.it.

A. Singh is with the Faculty of Electrical and Computer Engineering, Biomedical Engineering and Mathematical Sciences, University of Delaware, Newark, DE 19716, USA. E-mail address: absingh@udel.edu.
The chemical reaction scheme under investigation is the one reported in Fig. 1, and will be referred to in the sequel as scheme 0 . It consists of a substrate $S$ binding to an enzyme $E$ in order to form a complex $C$ (reaction 1) which in turn can reverse the binding (reaction 2) or can be transformed into a product $P$ (with the release of the enzyme $E$, reaction $3)$. The reaction scheme accounts for enzyme production (reaction 4) and degradation (reaction 5), product elimination (for instance due to its final utilization, reaction 6) and substrate production (reaction 7). With respect to the enzyme

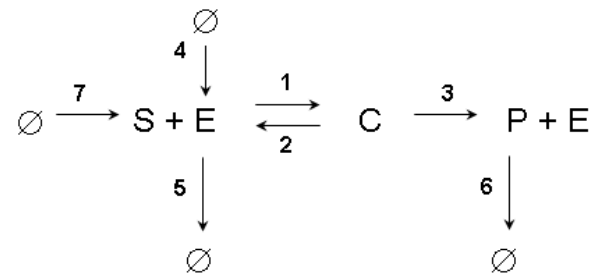

Fig. 1. Scheme 0: general reaction framework without feedback on the enzyme production

production, besides scheme 0 where there is no feedback regulation, two different schemes will be investigated: scheme $A$, where the enzyme production involves a negative feedback regulation from the product $P$ (Fig. 2) and scheme $B$, where the enzyme production is negatively regulated in feedback by the enzyme $E$ itself (Fig. 3). A similar reaction scheme

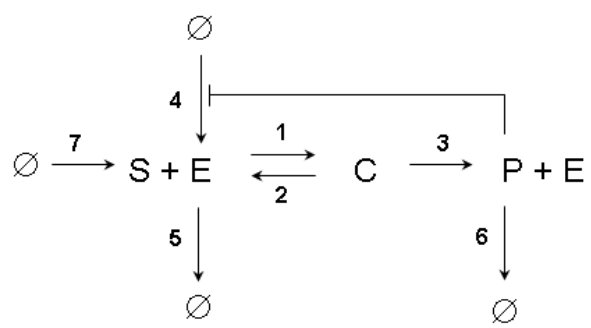

Fig. 2. Scheme A: enzyme production is negatively regulated by a feedback from the product

has been investigated also in [6], where only the feedback from the product had been considered (instead of the present two feedback schemes); besides, here, also the substrate production has been taken into account, with the molecule production (both for the substrate and the enzyme) occurring by means of bursts of molecules [7].

A proper way to quantify the metabolic noise involving the product fluctuations around its steady-state average value 


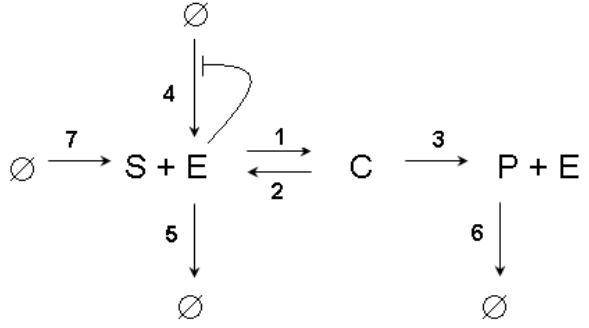

Fig. 3. Scheme B: enzyme production is negatively regulated by a feedback from the enzyme itself

is to exploit the stochastic approach based on the Chemical Master Equations (CME), providing a description of complex cellular processes much more accurate than the deterministic one [8]. The CME is capable to cope with fluctuations and chemical fluxes, to fit experimental data in the today widespread single cell experiments, and to capture and explain the deviation from Gaussianity observed in various gene expression experiments (such as stress or metabolic response, growth of the nuclear protein amount observed in senescent cells, and so on).

The main problem in dealing with $\mathrm{CME}$ is the curse of dimensionality which, in many cases, prevents from explicitly computing the solutions and thus requires implementing Monte Carlo methods (e.g. the Gillespie Stochastic Simulation Algorithm (SSA) [9]) to estimate the stationary distribution. Unfortunately, in the case of enzymatic reactions, the double time-scale of the reactions involved (binding/unbinding reactions occur on a shorter time-scale than product formation and enzyme production) makes exact SSA computationally demanding, since it gets stuck on thousands of binding/unbinding reactions for each birth of a product molecule [6].

In this paper, to cope with the high dimensionality of the system and the intrinsic double time-scale of the reaction network, which make practically unfeasible both the theoretical and the statistical computation of the CME solution, we resort to the Linear Noise Approximation [8], according to which the stationary distribution of the species' copy numbers is approximated by a multivariate Gaussian, with the average provided by the deterministic ODEs associated to the chemical reactions and the covariance matrix obtained by means of the linear approximation of the nonlinear terms of the ODE system. LNA is exploited to obtain a numerical evaluation of the metabolic noise. On top of that, the QuasiSteady-State Approximation (QSSA) is introduced [10], [11], which properly exploits the double time-scale of the system to reduce the complexity of the slow dynamics. Its validity is discussed by comparing the LNA numerical results for the original system to those for the reduced system coming from the QSSA. Moreover, the simplifications introduced by the QSSA enable the analytic computation of the variances of the species' copy numbers, thus allowing to explicitly correlate the metabolic noise of the product to the model parameters. Theory and simulations agree in highlighting the effective role of the negative feedback (in both schemes A and B) in reducing the random fluctuations, independently of the parameter setting. On the other hand, different choices of the system parameters make it so that sometimes feedback scheme A works better than feedback scheme B, while some other times the opposite occurs.

The paper is organized as follows. Section 2 reviews the stochastic approach adopted to model the three schemes under investigation. Section 3 is devoted to derive the firstorder moment equations, providing useful (exact) information on the steady-state average values of complex, enzyme and product. Section 4 deals with the LNA approach and numerical simulations are carried out. By exploiting the QSSA assumption, Section 5 provides the analytical expressions of the metabolic noise in the LNA framework. Conclusions follow.

\section{MATHEMATICAL MODEL OF CHEMICAL REACTION SCHEMES}

According to the standard stochastic approach to chemical reaction modeling [8], the state of the system is identified by the copy number of each involved species. Such quantities (grouped in vector form) will be denoted by small letters $\left[\begin{array}{llll}s & e & c & p\end{array}\right]^{T}$, while we employ the capital letters $S, E, C, P$ to denote the corresponding chemical species. The temporal evolution of a reaction network is described by a ContinuousTime Markov Process, where a state-dependent propensity $w_{j}$ is associated to each reaction $j$.

In the following, unless differently specified, the expected value of a random variable $x$ will be denoted by $\langle x\rangle$, while the steady-state average value of a stochastic process $x(t)$ will be denoted by $x^{\star}=\lim _{t \mapsto+\infty}\langle x(t)\rangle$.

\section{Scheme 0: no feedback}

Reactions $r_{1}$ and $r_{2}$ refer to standard binding/unbinding, formally defined as:

$$
\begin{aligned}
& \left.r_{1}\right) S+E \stackrel{1}{\longrightarrow} C \Longrightarrow s \mapsto s-1, e \mapsto e-1, c \mapsto c+1 \\
& \left.r_{2}\right) C \stackrel{2}{\longrightarrow} S+E \Longrightarrow s \mapsto s+1, e \mapsto e+1, c \mapsto c-1
\end{aligned}
$$

with propensities given by $w_{1}(s, e)=k_{1} s e, w_{2}(c)=k_{2} c$, respectively.

Reaction $r_{3}$ is formally similar to $r_{2}$, since it allows the disaggregation of complex $C$ by means of the product $P$ and the free enzyme $E$ :

$\left.r_{3}\right) \stackrel{3}{\longrightarrow} P+E$

with propensity $w_{3}(c)=k_{3} c$

Reactions $r_{4}$ refer to the enzyme production:

$\left.r_{4}\right) \emptyset \stackrel{4}{\longrightarrow} E \quad \Longrightarrow \quad e \mapsto e+N_{e}$

meaning that the production occurs in bursts of $N_{e} \in \mathbb{N}=$ $\{1,2, \ldots\}$ new molecules. As a matter of fact, the enzyme production is modeled by a set of infinite burst reactions, each referring to a different burst size in $\mathbb{N}$. Like in [7], [12] we assume that $N_{e}$ is a random variable with geometric distribution of mean $\left\langle N_{e}\right\rangle=\mu_{e}$ and second order moment $\left\langle N_{e}^{2}\right\rangle=\xi_{e}^{2}$, so that the propensity of a reaction of type $r_{4}$ producing $n_{e} \in \mathbb{N}$ enzyme copies is given by $w_{40}\left(n_{e}\right)=$ $k_{4} \mathbb{P}\left(N_{e}=n_{e}\right)$. The notation $w_{40}$ stresses the fact that we are dealing with reaction $r_{4}$, scheme 0 .

Reactions $r_{5}$ and $r_{6}$ are degradation/export clearance rates: 
$\begin{array}{lll}\left.r_{5}\right) E \stackrel{5}{\longrightarrow} \emptyset & \Longrightarrow & e \mapsto e-1 \\ \left.r_{6}\right) P \stackrel{6}{\longrightarrow} \emptyset \quad \Longrightarrow & p \mapsto p-1\end{array}$

with propensities $w_{5}(e)=k_{5} e, w_{6}(p)=k_{6} p$, respectively.

Reactions $r_{7}$ refer to the substrate production:

$\left.r_{7}\right) \emptyset \stackrel{7}{\longrightarrow} S \quad \Longrightarrow \quad s \mapsto s+N_{s}$

meaning that the production occurs in bursts of $N_{s} \in \mathbb{N}$ new molecules. Like for the enzyme production, also the substrate production is modeled by a set of infinite burst reactions, and $N_{s}$ is assumed to be a random variable with geometric distribution of mean $\left\langle N_{s}\right\rangle=\mu_{s}$ and second order moment $\left\langle N_{s}^{2}\right\rangle=\xi_{s}^{2}$, so that the propensity of a reaction of type $r_{7}$ producing $n_{s} \in \mathbb{N}$ substrate copies is given by $w_{7}\left(n_{s}\right)=k_{7} \mathbb{P}\left(N_{s}=n_{s}\right)$.

\section{Scheme A: feedback from the product $P$}

In order to account for the negative feedback from the product acting on the enzyme production, only the propensity of a reaction of type $r_{4}$ producing $n_{e} \in \mathbb{N}$ enzyme copies is modified into $w_{4 a}\left(p, n_{e}\right)=g(p) \mathbb{P}\left(N_{e}=n_{e}\right)$, with

$$
g(p)=\frac{\beta_{p}}{1+\left(p / \theta_{p}\right)^{h_{p}}}
$$

where $\beta_{p}$ provides the maximal propensity, $\theta_{p}$ is the repression threshold, required to reduce the propensity of $50 \%$, and $h_{p}$ is the Hill coefficient, providing the steepness of the sigmoidal Hill function $g$. Under the hypothesis of small noise fluctuations around the average steady-state copy number $p^{\star}$, the first-order approximation within the neighborhood of $p^{\star}$ will be considered [13], so that:

$$
g(p) \simeq g\left(p^{*}\right)+g^{\prime}\left(p^{*}\right)\left(p-p^{*}\right) .
$$

\section{Scheme B: feedback from the enzyme $E$}

Also in this case, in order to account for the negative selfregulation of the enzyme acting on its own production, only the propensity of a reaction of type $r_{4}$ producing $n_{e} \in \mathbb{N}$ enzyme copies is modified into $w_{4 b}\left(e, n_{e}\right)=f(e) \mathbb{P}\left(N_{e}=\right.$ $n_{e}$ ), with

$$
f(e)=\frac{\beta_{e}}{1+\left(e / \theta_{e}\right)^{h_{e}}}
$$

where $\beta_{e}, \theta_{e}$ and $h_{e}$ play the same role of the corresponding parameters in (1). Also in this case, under the hypothesis of small noise fluctuations around the average steady-state copy number $e^{\star}$, the first-order approximation within the neighborhood of $e^{\star}$ will be considered [13], so that:

$$
f(e) \simeq f\left(e^{*}\right)+f^{\prime}\left(e^{*}\right)\left(e-e^{*}\right) .
$$

\section{Metabolic noise estimation}

Referring to the product $P$, we define the corresponding metabolic noise by means of the square of the coefficient of variation $C V_{P}^{2}$ computed by the ratio:

$$
C V_{P}^{2}=\sigma_{P}^{2} /\left(p^{\star}\right)^{2}
$$

where $\sigma_{P}^{2}$ and $p^{\star}$ are the steady-state values for variance and mean of the marginal distribution of the product $P$ copy number. The proper way to compute $C V_{P}^{2}$ is by means of the Chemical Master Equation (CME) [8]. Unfortunately, to solve the CME is a hard nut to crack: different methods are available to compute exact or approximate solutions (see, e.g. [14], [15], [16]), as well as statistical approaches (such as the Stochastic Simulation Algorithm, SSA, [9]). In the following sections, the computation of the metabolic noise will be carried out by means of the Linear Noise Approximation (LNA) [8], which enables to obtain an analytical expression of the variances of the species' copy numbers. Such quantities will be computed under the Quasi-Steady-State Approximation (QSSA) assumption, in order to shorten the equations and to highlight explicit correlations between the model parameters and the metabolic noise.

\section{FIRST ORDER MOMENT EQUATIONS}

According to [17], we define $\eta=\left[\begin{array}{llll}\eta_{s} & \eta_{e} & \eta_{c} & \eta_{p}\end{array}\right]^{T}:=$ $\left[\begin{array}{llll}\langle s\rangle & \langle e\rangle & \langle c\rangle & \langle p\rangle\end{array}\right]^{T}$ and we can write the first order moment equations in the following fashion for the three schemes:

$$
\dot{\eta}(t)=A_{1} \eta(t)+b_{1}+\Gamma_{1}\langle s e\rangle
$$

with:

$$
\begin{aligned}
A_{1} & =\left[\begin{array}{cccc}
0 & 0 & k_{2} & 0 \\
0 & \alpha_{22}\left(e^{\star}\right) \mu_{e}-k_{5} & k_{2}+k_{3} & \alpha_{24}\left(p^{\star}\right) \mu_{e} \\
0 & 0 & -\left(k_{2}+k_{3}\right) & 0 \\
0 & 0 & k_{3} & -k_{6}
\end{array}\right], \\
b_{1} & =\left[k_{7} \mu_{s}, \delta\left(e^{\star}, p^{\star}\right) \mu_{e}, 0,0\right]^{T}, \quad \Gamma_{1}=\left[-k_{1},-k_{1}, k_{1}, 0\right]^{T},
\end{aligned}
$$

where

$$
\begin{aligned}
& \alpha_{22}\left(e^{\star}\right)= \begin{cases}0 & \text { in scheme } 0 \text { and in scheme A, } \\
f^{\prime}\left(e^{\star}\right) & \text { in scheme B, }\end{cases} \\
& \alpha_{24}\left(p^{\star}\right)= \begin{cases}0 & \text { in scheme } 0 \text { and in scheme B, } \\
g^{\prime}\left(p^{\star}\right) & \text { in scheme A, }\end{cases}
\end{aligned}
$$$$
\delta\left(e^{\star}, p^{\star}\right)= \begin{cases}k_{4} & \text { in scheme 0, } \\ g\left(p^{\star}\right)-g^{\prime}\left(p^{\star}\right) p^{\star} & \text { in scheme A, } \\ f\left(e^{\star}\right)-f^{\prime}\left(e^{\star}\right) e^{\star} & \text { in scheme B. }\end{cases}
$$

It is worth noting that, although Eq.(6) is not in closed form (and therefore it cannot be solved), it is useful to infer information about the steady state without any approximation. Hence, solving (6) at the equilibrium $(\dot{\eta}(t)=0)$ leads to the following values for product and the complex steady states:

$$
c^{\star}=k_{7} \mu_{s} / k_{3} \quad p^{\star}=k_{7} \mu_{s} / k_{6},
$$

while the enzyme steady state satisfies the following equation:

$$
e^{\star}=\frac{\left(\delta\left(e^{\star}, p^{\star}\right)+\alpha_{24}\left(p^{\star}\right) p^{\star}\right) \mu_{e}}{k_{5}-\alpha_{22}\left(e^{\star}\right) \mu_{e}} .
$$

Eq.(8) provides an explicit expression for the average steadystate copy number of the enzyme for schemes 0 and A:

$$
e_{0}^{\star}=k_{4} \mu_{e} / k_{5} \quad e_{A}^{\star}=g\left(p^{\star}\right) \mu_{e} / k_{5}
$$

whilst, for scheme $B$, it only ensures the existence of a unique solution satisfying:

$$
e_{B}^{\star}=f\left(e^{\star}\right) \mu_{e} / k_{5} .
$$

It can be easily verified that, for any values of the parameters, the product and the complex average steady state values 
are independent of the chosen scheme, while the enzyme average stationary value varies, in general, from scheme to scheme. So, in order to make a fair comparison among the three schemes, the following assumptions are adopted for the setting of the Hill functions $f, g$ defined in (1), (3), to obtain the same enzyme average stationary value:

- the same Hill coefficient is chosen: $h_{e}=h_{p}=h$;

- the thresholds are set equal to the corresponding steady state values: $\theta_{p}=p^{\star}, \theta_{e}=e^{\star}$. This allows to easily find the steady state value for the enzyme, since $g\left(p^{\star}\right)=$ $\left(\beta_{p} / 2\right)$ and $f\left(e^{\star}\right)=\left(\beta_{e} / 2\right)$ :

$$
e_{A}^{\star}=\beta_{p} \mu_{e} /\left(2 k_{5}\right), \quad e_{B}^{\star}=\beta_{e} \mu_{e} /\left(2 k_{5}\right) ;
$$

- parameters $\beta_{p}, \beta_{e}$ are chosen to obtain the same steady state value for the enzyme: $\beta_{e}=\beta_{p}=2 k_{4}$.

As a consequence, according to (2), (4) and to the aforementioned assumptions, we have:

$$
\begin{aligned}
& w_{4 a}\left(p, n_{e}\right)=\left(k_{4}+g^{\prime}\left(p^{\star}\right)\left(p-p^{\star}\right)\right) \mathbb{P}\left(N_{e}=n_{e}\right) \\
& w_{4 b}\left(e, n_{e}\right)=\left(k_{4}+f^{\prime}\left(e^{\star}\right)\left(e-e^{\star}\right)\right) \mathbb{P}\left(N_{e}=n_{e}\right)
\end{aligned}
$$

with:

$$
\begin{gathered}
g^{\prime}\left(p^{\star}\right)=-h k_{4} k_{6} /\left(2 k_{7} \mu_{s}\right), \quad f^{\prime}\left(e^{\star}\right)=-h k_{5} /\left(2 \mu_{e}\right) . \\
\text { IV. LINEAR NOISE APPROXIMATION (LNA) } \\
\text { FOR ENZYMATIC KINETICS }
\end{gathered}
$$

The LNA approximating assumption is that molecular fluctuations provide small deviations around the stationary average values. According to this hypothesis, the LNA approximates the stationary distribution of the copy numbers by a multivariate Gaussian with the average value computed by means of the steady-state solution of the deterministic ODE system associated with the chemical reactions, written with respect to the concentrations (instead of copy numbers) according to the mass action law [8]. To this end, define $\gamma=\left[\begin{array}{llll}\gamma_{s} & \gamma_{e} & \gamma_{c} & \gamma_{p}\end{array}\right]^{T}$ as the vector of concentrations associated to the vector of copy numbers $\left[\begin{array}{llll}s & e & c & p\end{array}\right]^{T}$, and let $V$ be the (constant) distribution volume; the ODE concentration system can be written in compact form as:

$$
\dot{\gamma}(t)=N \mathcal{V}(\gamma(t)) \text {, }
$$

with $N$ being the stoichiometric matrix associated to the considered reactions and species, and with vector $\mathcal{V}$ collecting the macroscopic reaction rates. In order to properly account for the many (infinite) burst reactions of type $r_{4}$ and $r_{7}$ providing substrate and enzyme production, the following notation is adopted:

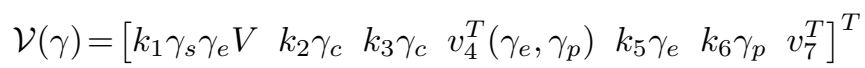

with $v_{4}\left(\gamma_{e}, \gamma_{p}\right)=\rho\left(\gamma_{e}, \gamma_{p}\right)\left[\mathbb{P}\left(N_{e}=1\right) \mathbb{P}\left(N_{e}=2\right) \cdots\right]^{T}$ and $v_{7}=k_{7}\left[\mathbb{P}\left(N_{s}=1\right) \quad \mathbb{P}\left(N_{s}=2\right) \quad \cdots\right]^{T}$ being vectors of infinite elements, and $\rho\left(\gamma_{e}, \gamma_{p}\right)=\alpha_{22}\left(e^{\star}\right) \gamma_{e}+$ $\alpha_{24}\left(p^{\star}\right) \gamma_{p}+\frac{\delta\left(e^{\star}, p^{\star}\right)}{V}$. Matrix $N$ is defined as:

$$
N=\left[\begin{array}{ccccccc}
-1 & 1 & 0 & \mathbf{0}^{T} & 0 & 0 & \mathbf{n}^{T} \\
-1 & 1 & 1 & \mathbf{n}^{T} & -1 & 0 & \mathbf{0}^{T} \\
1 & -1 & -1 & \mathbf{0}^{T} & 0 & 0 & \mathbf{0}^{T} \\
0 & 0 & 1 & \mathbf{0}^{T} & 0 & -1 & \mathbf{0}^{T}
\end{array}\right]
$$

with $\mathbf{0}^{T}$ being an infinite row of all zero elements and $\mathbf{n}^{T}=$ $\left[\begin{array}{lll}1 & 2 & \cdots\end{array}\right]^{T}$ being a row vector aggregating the ordered sequence of integers.

The adoption of infinite-dimensional vectors and matrices is only a matter of convenience of notation, since the product in (14) provides a finite-dimensional ODE system, whose state vector $\gamma$ could also be derived from the first order moment equations (6) after: (i) closing the first-order equations (see e.g. [15]) by approximating $\langle s e\rangle=\eta_{s} \eta_{e}$, (ii) accounting for the conversion from average copy number to concentration $\gamma=\eta / V$ :

$$
\dot{\gamma}(t)=A_{1} \gamma(t)+b_{1} / V+V \Gamma_{1} \gamma_{s}(t) \gamma_{e}(t) \text {. }
$$

It is worth noting that the steady-state solutions computed from (16) in terms of enzyme, complex and product correspond to the steady-state solutions coming from the firstorder moment equations (6), so they are not approximated values. Moreover, (16) allows to compute the steady-state (approximate) value also for the substrate, which could not be computed from (6). This value will be denoted by $s^{\star}$ (with a little abuse of notation, since $s^{\star}$ in the following equation is an approximation of the average steady-state value):

$s^{\star}=V \gamma_{s}^{\star}=k_{5} k_{7}\left(k_{2}+k_{3}\right) \mu_{s} /\left(k_{1} k_{3} k_{4} \mu_{e}\right) \simeq \lim _{t \mapsto+\infty}\langle s(t)\rangle$.

The covariance matrix $\Sigma$ associated to the copy number fluctuations around the equilibrium solution of (16) is the positive-definite solution of the Lyapunov equation [8]:

$$
J \Sigma+\Sigma J^{T}+V B B^{T}=0
$$

with

$$
J=N \cdot(d \mathcal{V} / d \gamma)_{\left.\right|_{\gamma^{\star}}} \quad B B^{T}=N \operatorname{diag}\left\{\mathcal{V}\left(\gamma^{\star}\right)\right\} N^{T} .
$$

Note that, when computing $B B^{T}$, the second order moments $\xi_{s}^{2}, \xi_{e}^{2}$ of the geometric distributions for the production burst sizes are exploited. As a further remark, the volume $V$ affects neither the computation of the steady-state of (16) (in terms of copy number), nor the computation of $\Sigma$ in (17).

Simulations have been carried out by exploiting the following set of nominal model parameters (most of which taken from [6]): $k_{1}=1 \mathrm{~s}^{-1}$ molecule ${ }^{-1}, k_{2}=28300 \mathrm{~s}^{-1}$, $k_{3}=3.2 s^{-1}, k_{4}=0.01 s^{-1}, k_{5}=10^{-3} \mathrm{~s}^{-1}, k_{6}=0.02 s^{-1}$, $k_{7}=1.2 s^{-1}, \mu_{e}=3$ molecule, $\mu_{s}=5.67$ molecule, $\xi_{e}^{2}=$ 21 molecule $^{2}, \xi_{s}^{2}=69.89$ molecule $^{2}, h_{e}=h_{s}=h=5$, with $\beta_{p}=\beta_{e}=2 k_{4}$ and $\theta_{p}=p^{\star}, \theta_{e}=e^{\star}$ (see previous section). Then, from (7), (9), (11), one gets $e^{\star}=30, c^{\star}=2.125$, $p^{\star}=340$, with $e^{\star}$ sharing the same value for the three schemes.

Results obtained from the LNA according to different values of $k_{4}$ and of $h_{p}=h_{e}=h$ (strength of the feedback) are summarized in Tables I and II, respectively. Note that the noise in scheme 0 is always higher than the one in the feedback schemes A and B, but the type of feedback providing the best performance depends on the particular choice of the parameters. 
TABLE I

METABOLIC NOISE FOR DIFFERENT VALUES OF PARAMETER $k_{4}$

\begin{tabular}{|c|c|c|c|}
\hline$C V_{P}^{2}$ & $\begin{array}{c}k_{4}=10^{-3} \\
e^{\star}=3\end{array}$ & $\begin{array}{c}k_{4}=10^{-2} \\
e^{\star}=30\end{array}$ & $\begin{array}{c}k_{4}=10^{-1} \\
e^{\star}=300\end{array}$ \\
\hline 0 & $5.64 \cdot 10^{-1}$ & $2.86 \cdot 10^{-2}$ & $1.35 \cdot 10^{-2}$ \\
\hline $\mathrm{A}$ & $1.99 \cdot 10^{-1}$ & $1.92 \cdot 10^{-2}$ & $1.27 \cdot 10^{-2}$ \\
\hline $\mathrm{B}$ & $1.86 \cdot 10^{-1}$ & $1.87 \cdot 10^{-2}$ & $1.35 \cdot 10^{-2}$ \\
\hline
\end{tabular}

TABLE II

METABOLIC NOISE FOR DIFFERENT VALUES OF PARAMETERS $h_{p}=h_{e}$

\begin{tabular}{|c|c|c|c|}
\hline$C V_{P}^{2}$ & $h_{p}=h_{e}=1$ & $h_{p}=h_{e}=5$ & $h_{p}=h_{e}=10$ \\
\hline 0 & $2.86 \cdot 10^{-2}$ & $2.86 \cdot 10^{-2}$ & $2.86 \cdot 10^{-2}$ \\
\hline $\mathrm{A}$ & $2.60 \cdot 10^{-2}$ & $1.92 \cdot 10^{-2}$ & $1.47 \cdot 10^{-2}$ \\
\hline $\mathrm{B}$ & $2.58 \cdot 10^{-2}$ & $1.87 \cdot 10^{-2}$ & $1.43 \cdot 10^{-2}$ \\
\hline
\end{tabular}

\section{COMPUTATIONS IN THE QSSA FRAMEWORK}

In order to write an analytical expression for the metabolic noise affecting the product $P$, the LNA will be applied to the standard Quasi-Steady-State Approximation (QSSA) of the deterministic equations given by (16). The QSSA is a widespread approach adopted to reduce the computational complexity in the presence of a typical fast/slow time-scale of enzymatic reactions; see e.g. [11] and references therein for an exhaustive review of advantages and limitations of such an approach, which substantially exploits the faster dynamics of complex $C$, supposed to be negligible (i.e. $\dot{\gamma}_{c}=0$ ) with respect to the other players' dynamics. This approximation clearly does not affect the steady-states of (16), but allows to simplify the corresponding Lyapunov equation in (17)-(18) where, after some computations, one gets:

$$
J=\left[\begin{array}{ccc}
-\frac{k_{1} k_{3} e^{\star}}{k_{2}+k_{3}} & -\frac{k_{1} k_{3} s^{\star}}{k_{2}+k_{3}} & 0 \\
0 & \rho_{e}^{\star} \mu_{e}-k_{5} & \rho_{p}^{\star} \mu_{e} \\
\frac{k_{1} k_{3} e^{\star}}{k_{2}+k_{3}} & \frac{k_{1} k_{3} s^{\star}}{k_{2}+k_{3}} & -k_{6}
\end{array}\right]
$$

with $\rho_{e}^{\star}=\left.\frac{\partial \rho}{\partial e}\right|_{\left(e^{\star}, p^{\star}\right)}=\alpha_{22}\left(e^{\star}\right), \rho_{p}^{\star}=\left.\frac{\partial \rho}{\partial p}\right|_{\left(e^{\star}, p^{\star}\right)}=$ $\alpha_{24}\left(p^{\star}\right)$, and

$$
B B^{T}=\frac{1}{V}\left[\begin{array}{ccc}
k_{7}\left(\mu_{s}+\xi_{s}^{2}\right) & 0 & -k_{7} \mu_{s} \\
0 & k_{5} e^{\star}\left(\xi_{e}^{2}+\mu_{e}\right) / \mu_{e} & 0 \\
-k_{7} \mu_{s} & 0 & 2 k_{7} \mu_{s}
\end{array}\right]
$$

where the identities $\rho\left(\gamma_{e}^{\star}, \gamma_{p}^{\star}\right)=\frac{k_{5} e^{\star}}{V \mu_{e}}=\frac{k_{4}}{V}$ coming from (8), (9) have been exploited.

By solving the Lyapunov equation (17), with $J$ and $B B^{T}$ given by (19) and (20), after some computations, we obtain the following results:

$$
\sigma_{P}^{2}=\frac{\frac{k_{7} \mu_{s}}{k_{6}}\left(k_{6}+\frac{\mu_{s}+\xi_{s}^{2}}{2 \mu_{s}} \frac{k_{1} k_{3} e^{\star}}{k_{2}+k_{3}}+\frac{k_{6} k_{7} \mu_{s}\left(k_{5} \frac{\mu_{e}+\xi_{e}^{2}}{2 \mu_{e}}-\rho_{p}^{\star} \mu_{e}\right)}{\Xi\left(e^{\star}\right)}\right)}{k_{6}+\frac{k_{1} k_{3} e^{\star}}{k_{2}+k_{3}}-\rho_{p}^{\star} k_{7} \mu_{s} \mu_{e}\left(\frac{k_{5}+k_{6}-\rho_{e}^{\star} \mu_{e}+\frac{k_{1} k_{3} e^{\star}}{k_{2}+k_{3}}}{\Xi\left(e^{\star}\right)}\right)}
$$

with $\Xi\left(e^{\star}\right)=e^{\star}\left(k_{5}+k_{6}-\rho_{e}^{\star} \mu_{e}\right)\left(k_{5}+\frac{k_{1} k_{3} e^{\star}}{k_{2}+k_{3}}-\rho_{e}^{\star} \mu_{e}\right)$. Note that (21) is valid for the three schemes under investigation. In more details, accounting for the scheme without feedback regulation, since $\rho_{e}^{\star}=\rho_{p}^{\star}=0$, Eq.(21) reduces to:

$$
\sigma_{P, 0}^{2}=\frac{\frac{k_{7} \mu_{s}}{k_{6}}\left(k_{6}+\frac{\mu_{s}+\xi_{s}^{2}}{2 \mu_{s}} \frac{k_{1} k_{3} e^{\star}}{k_{2}+k_{3}}+\frac{k_{5} k_{6} k_{7} \mu_{s}\left(\mu_{e}+\xi_{e}^{2}\right)}{2 \mu_{e} \Xi_{0}\left(e^{\star}\right)}\right)}{k_{6}+\frac{k_{1} k_{3} e^{\star}}{k_{2}+k_{3}}}
$$

with $\Xi_{0}\left(e^{\star}\right)=e^{\star}\left(k_{5}+k_{6}\right)\left(k_{5}+\frac{k_{1} k_{3} e^{\star}}{k_{2}+k_{3}}\right)$. For the other schemes, we obtain:

$$
\sigma_{P, A}^{2}=\frac{\frac{k_{7} \mu_{s}}{k_{6}}\left(k_{6}+\frac{\mu_{s}+\xi_{s}^{2}}{2 \mu_{s}} \frac{k_{1} k_{3} e^{\star}}{k_{2}+k_{3}}+\frac{k_{6} k_{7} \mu_{s}\left(k_{5} \frac{\mu_{e}+\xi_{e}^{2}}{2 \mu_{e}}-\rho_{p}^{\star} \mu_{e}\right)}{\Xi_{a}\left(e^{\star}\right)}\right)}{k_{6}+\frac{k_{1} k_{3} e^{\star}}{k_{2}+k_{3}}-\rho_{p}^{\star} k_{7} \mu_{s} \mu_{e}\left(\frac{k_{5}+k_{6}+\frac{k_{1} k_{3} e^{\star}}{k_{2}+k_{3}}}{\Xi_{A}\left(e^{\star}\right)}\right)}
$$

with $\rho_{p}^{\star}=g^{\prime}\left(p^{\star}\right)$ and $\Xi_{A}\left(e^{\star}\right)=\Xi_{0}\left(e^{\star}\right)$, and

$$
\sigma_{P, B}^{2}=\frac{\frac{k_{7} \mu_{s}}{k_{6}}\left(k_{6}+\frac{\mu_{s}+\xi_{s}^{2}}{2 \mu_{s}} \frac{k_{1} k_{3} e^{\star}}{k_{2}+k_{3}}+\frac{k_{5} k_{6} k_{7} \mu_{s}\left(\mu_{e}+\xi_{e}^{2}\right)}{2 \mu_{e} \Xi_{B}\left(e^{\star}\right)}\right)}{k_{6}+\frac{k_{1} k_{3} e^{\star}}{k_{2}+k_{3}}}
$$

with $\rho_{e}^{\star}=f^{\prime}\left(e^{\star}\right)$, and $\Xi_{B}\left(e^{\star}\right)=e^{\star}\left(k_{5}+k_{6}-\right.$ $\left.f^{\prime}\left(e^{\star}\right) \mu_{e}\right)\left(k_{5}+\frac{k_{1} k_{3} e^{\star}}{k_{2}+k_{3}}-f^{\prime}\left(e^{\star}\right) \mu_{e}\right)$.

It is readily seen that $\sigma_{P, 0}^{2}$ differs from $\sigma_{P, B}^{2}$ only for $\Xi_{0}\left(e^{\star}\right)$ being replaced by $\Xi_{B}\left(e^{\star}\right)$. Moreover, since $f^{\prime}\left(e^{\star}\right)<$ 0 from the derivative of the inhibitory Hill function in (13), one obtains, for any parameter setting:

$$
\Xi_{0}\left(e^{\star}\right)<\Xi_{B}\left(e^{\star}\right) \quad \Longrightarrow \quad \sigma_{P, B}^{2}<\sigma_{P, 0}^{2}
$$

implying $C V_{P, B}<C V_{P, 0}$. An analogous result occurs for the comparison between the metabolic noise in scheme 0 and in scheme A, according to the following Lemma, whose proof we omit for lack of space.

Lemma 1: $C V_{P, 0}^{2}>C V_{P, A}^{2}$ for any parameter setting.

The use of analytical expressions for the variances in (22), (23) and (24) allows to investigate how the metabolic noise varies with respect to some crucial parameters, without involving numerical simulations.

In Fig. 4 we report $C V_{P}^{2}$ as a function of parameter $k_{4}$ (keeping all the other parameters fixed at the nominal values). The analytical expressions are compared to numerical samples (circles in the figure) taken by means of the LNA of the original system (without QSSA), to show the correct computations related to the QSSA and to validate the related assumption. It clearly appears that by increasing the value of $k_{4}$, the three schemes converge to the same behavior. It means that, the higher $k_{4}$ is, the less effective the feedback is in reducing the metabolic noise. Indeed:

$\lim _{k_{4} \mapsto+\infty} C V_{P, 0}^{2}=\lim _{k_{4} \mapsto+\infty} C V_{P, A}^{2}=\lim _{k_{4} \mapsto+\infty} C V_{P, B}^{2}=\frac{k_{6}\left(\mu_{s}+\xi_{s}^{2}\right)}{2 k_{7} \mu_{s}^{2}}$.

On the other hand, by decreasing $k_{4}$, the three curves detach from each other, highlighting the noise reduction properties of the feedback. Finally, Fig. 4 shows also that the two different feedback schemes may be one more effective than the other, according to a different setting of $k_{4}$. This fact is appreciable also by looking at data in Table I, where the LNA is applied without QSSA. 


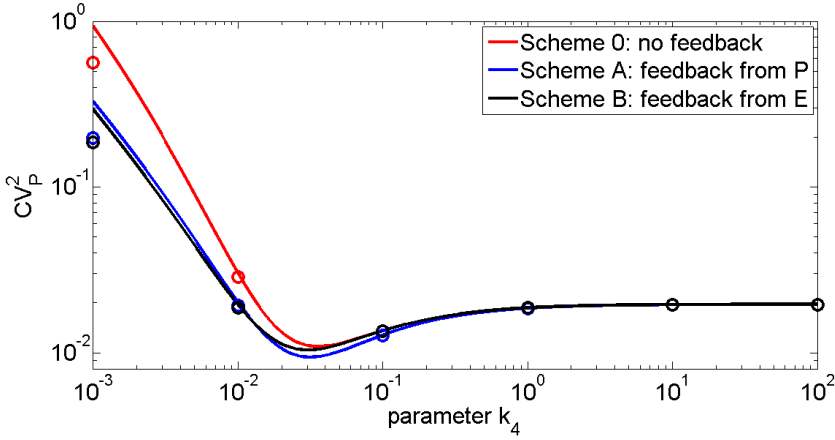

Fig. 4. Metabolic noise as a function of parameter $k_{4}$. The circles refer to numerical values achieved by means of LNA on the original system (without QSSA).

Fig. 5 reports $C V_{P}^{2}$ as a function of the Hill coefficient $h$ of the sigmoidal functions (the strength of the negative feedback). Two distinct scenarios have been accounted for in Fig. 5: the continuous lines refer to the case $k_{4}=0.01$, whereas the dashed lines refer to the case $k_{4}=0.03$. For

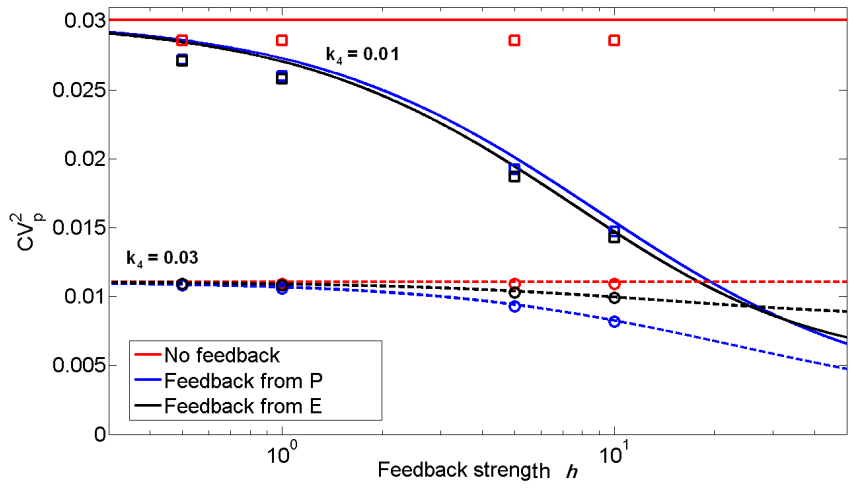

Fig. 5. Metabolic noise as a function of the Hill coefficient $h$. Continuous lines refer to $k_{4}=0.01$, whereas dashed lines refer to $k_{4}=0.03$. Markers (squares for $k_{4}=0.01$ and circles for $k_{4}=0.03$ ) refer to numerical values achieved by means of LNA on the original system (without QSSA).

$h \mapsto 0$, the strength of the feedback reduces more and more and, as a matter of fact, both feedback schemes tend to behave like the scheme without feedback (scheme 0 ) in both scenarios. This fact can be easily anticipated by the analytic expressions, since:

$$
\begin{aligned}
& \lim _{h \mapsto 0^{+}} \rho_{p}^{\star}=\lim _{h \mapsto 0^{+}} \rho_{e}^{\star}=0 \quad \Longrightarrow \sigma_{P, A}^{2} \mapsto \sigma_{P, 0}^{2} \\
& \lim _{h \mapsto 0^{+}} \Xi_{B}\left(e^{\star}\right)=\lim _{h \mapsto 0^{+}} \Xi_{0}\left(e^{\star}\right) \Longrightarrow \begin{array}{c}
\sigma_{P, A}^{2} \\
\sigma_{P, B}^{2} \mapsto \sigma_{P, 0}^{2}
\end{array}
\end{aligned}
$$

On the other hand, by increasing $h$, the feedback schemes $\mathrm{A}$ and $\mathrm{B}$ become more and more effective in noise reduction, with respect to the scheme without feedback. What appears from this figure is that different values of $k_{4}$ may well influence which of the two feedback schemes works better, in conjunction with reasonable values of the Hill coefficient.

\section{CONCLUSIONS}

In this work, we have presented a comparison between different basic schemes for the noise reduction in enzymatic reactions, where the noise is evaluated in terms of coefficient of variation of the reaction product. The case where the enzyme production is not regulated is outperformed by the two regulation schemes (product-feedback and enzymefeedback), as shown by evaluating the noise by means of numerical simulation in the Linear Noise Approximation (LNA) framework. Exploiting the assumption of double time-scale separation (QSSA), approximate analytical computations provide theoretical evidence for the experimental results. However, theory and simulations do not allow to conclude with certainty about which feedback scheme is the most effective, and further investigations are currently under study.

\section{ACKNOWLEDGEMENTS}

AS is supported by the National Science Foundation Grant DMS-1312926, University of Delaware Research Foundation (UDRF) and Oak Ridge Associated Universities (ORAU).

\section{REFERENCES}

[1] V. Henri, Lois generales de l'action des diastases, Librairie Scientifique A. Hermann, 1903.

[2] L. Michaelis, M. Menten, Kinetics of invertase action, Biochem. Z, 49, 333-369, 1913.

[3] A. Zaslaver, A. Mayo, R. Rosenberg, P. Bashkin, H. Sberro, M. Tsalyuk, M. Surette, U. Alon, Just-in-time transcription program in metabolic pathways, Nat. Genet. 36, 486-491, 2004.

[4] U. Alon, An Introduction to Systems Biology: Design Principles of Biological Circuits, Chapman and Hall/CRC, 2006.

[5] D.H. Calhoun, G.W. Hatfield, Autoregulation: a role for a biosynthetic enzyme in the control of gene expression, Proceedings of the National Academy of Sciences 70.10 (1973): 2757-2761.

[6] Oyarzun, D.A, Lugagne, J.-B., Stan, G.-B.V., Noise propagation in synthetic gene circuits for metabolic control, ACS Synthetic Biology, 2014.

[7] M. Soltani, C. Vargas, N. Kumar, R. Kulkarni, A. Singh, Conditional moment closure approximations in genetic negative feedback circuits. to appear on the Proc. American Control Conference (ACC), 2015.

[8] N.G. van Kampen, Stochastic Processes in Physics and Chemistry, North Holland, third edition, 2007.

[9] D. T. Gillespie, Exact Stochastic Simulation of Coupled Chemical Reactions, The Journal of Physical Chemistry 81(25), 2340-2361, 1977.

[10] L. Segel, On the validity of the steady state assumption of enzyme kinetics, Bull. Math. Biol. 50, 579-593, 1988.

[11] A.M. Bersani, E. Bersani, L. Mastroeni, Deterministic and stochastic models of enzymatic networks - applications to pharmaceutical research, Computers and Mathematics with Applications 55, 879-888, 2008.

[12] I. Golding, J. Paulsson, S. Zawilski, E. Cox, Real-time kinetics of gene activity in individual bacteria, Cell 123, 1025-1036, 2005.

[13] A. Singh, J.P. Hespanha, Optimal feedback strength for noise suppression in autoregulatory gene networks, Biophysical Journal 96, 40134023, 2009.

[14] B. Munsky and M. Khammash, The finite state projection approach for the analysis of stochastic noise in gene networks, IEEE Trans. Autom. Control, Special Issue on Systems Biology, 201-214, 2008.

[15] A. Singh, J.P. Hespanha, Approximate moment dynamics for chemically reacting systems, IEEE Transactions on Automatic Control 56, 414-418, 2011

[16] A. Borri, F. Carravetta, G. Mavelli, P. Palumbo, Some Results on the Structural Properties and the Solution of the Chemical Master Equation, Proceedings of the 2013 American Control Conference (ACC 2013), Washington, DC, USA, pp. 3777-3782, 2013.

[17] J.P. Hespanha, A. Singh, Stochastic models for chemically reacting systems using polynomial stochastic hybrid systems, Int. J. of Robust and Nonlinear Control 15, 669-689, 2005. 